Article

\title{
Geriatric Nutritional Risk Index as a Screening Tool to Identify Patients with Malnutrition at a High Risk of In-Hospital Mortality among Elderly Patients with Femoral Fractures-A Retrospective Study in a Level I Trauma Center
}

\author{
Wei-Ti Su ${ }^{1,+}$, Shao-Chun $\mathrm{Wu}^{2,+}{ }^{1}$, Chun-Ying Huang ${ }^{1}$, Sheng-En Chou ${ }^{1}$, Ching-Hua Tsai ${ }^{1}$, \\ Chi $\mathrm{Li}^{1}{ }^{1}$, Shiun-Yuan Hsu ${ }^{1}$ and Ching-Hua Hsieh ${ }^{3, *(1)}$ \\ 1 Department of Trauma Surgery, Kaohsiung Chang Gung Memorial Hospital, Chang Gung University \\ College of Medicine, Kaohsiung 803, Taiwan; s101132@adm.cgmh.org.tw (W.-T.S.); \\ junyinhaung@yahoo.com.tw (C.-Y.H.); athenechou@gmail.com (S.-E.C.); \\ tsai1737@cloud.cgmh.org.tw (C.-H.T.); foocollie7@gmail.com (C.L.); ah.lucy@hotmail.com (S.-Y.H.) \\ 2 Department of Anesthesiology, Kaohsiung Chang Gung Memorial Hospital, Chang Gung University College \\ of Medicine, Kaohsiung 803, Taiwan; shaochunwu@gmail.com \\ 3 Department of Plastic Surgery, Kaohsiung Chang Gung Memorial Hospital, Chang Gung University College \\ of Medicine, Kaohsiung 803, Taiwan \\ * Correspondence: m93chinghua@gmail.com \\ + These authors contributed equally to this work.
}

Received: 12 October 2020; Accepted: 27 November 2020; Published: 30 November 2020

\begin{abstract}
Background: Malnutrition is frequently underdiagnosed in geriatric patients and is considered to be a contributing factor for worse outcomes during hospitalization. In addition, elderly patients who undergo trauma are often malnourished at the time of incurring fractures. The geriatric nutritional risk index (GNRI), calculated based on the serum albumin level and the ratio of present body weight to ideal body weight, was proposed for the assessment of the nutritional status of elderly patients with various illnesses. This study aimed to investigate whether the GNRI has a prognostic value that links the nutritional status and mortality outcomes of elderly patients who have previously undergone trauma with femoral fractures. Methods: From January 1, 2009 to December 31, 2019, a total of 678 elderly patients with femoral fractures were categorized into four nutritional risk groups: a major-risk group (GNRI < 82; group 1, $n=127$ ), moderate-risk group (GNRI 82-92; group 2, $n=179$ ), low-risk group (GNRI 92-98; group 3, $n=123$ ), and no-risk group (GNRI > 98; group 4, $n=249$ ). To minimize the confounding effects of sex, age, preexisting comorbidities, and injury severity of patients on outcome measurements, propensity score-matched patient cohorts were created to assess the impact of patients being in different nutritional risk groups on the in-hospital mortality outcomes against the no-risk group. Results: The patients in groups 1-3 were significantly older and presented a significantly lower body mass index and lower serum albumin levels than those in group 4. Compared with patients in group $4(3.6 \%)$, a significantly higher mortality rate was found in the patients in group $1(17.3 \%, p<0.001)$, but not in those in group $2(6.7 \%)$ or group $3(2.4 \%)$. The study of propensity score-matched patient cohorts provided similar results; group 1 patients had significantly higher odds of mortality than group 4 patients (odds ratio, 6.3; 95\% confidence interval, 1.34-29.37; $p=0.009$ ), but there were no significant differences in mortality risks among patients in groups 2 and 3 compared with those in group 4 . Conclusions: This preliminary study suggested that the GNRI may be used as a screening tool to identify patients with malnutrition at a high risk of mortality among elderly patients with femoral fractures. A prospective study is needed to validate the suggestion.
\end{abstract}


Keywords: elderly; femoral bone fracture; malnutrition; mortality; geriatric nutritional risk index; trauma

\section{Background}

Elderly individuals are at the greatest risk of a fall accidents [1,2] and their consequences, such as fractures, immobility, and sometimes even death [3-7]. In addition to pain, a femoral fracture results in a curtailment of physical activity, increased dependence, and rapid worsening of the health status. In addition, older people with femoral fractures are often malnourished at the time of fracture, and subsequently have poor food intakes. Malnutrition is frequently underdiagnosed in geriatric patients [8] and is considered to be a contributing factor for worse outcomes during hospitalization [9-11] or after fracture surgery [12,13]. Therefore, a screening tool to identify patients with a high risk of malnutrition is important while caring for elderly patients with femoral fractures. Over 70 tools have been proposed for assessing nutritional status, 21 of which were designed for use in elderly patients [14]. However, no tool is currently considered the gold standard for nutritional assessment [15]. Tools such as the Subjective Global Assessment, Nutritional Risk Screening (NRS), and Mini Nutritional Assessment (MNA) showed a good correlation with the overall picture of malnutrition in geriatric patients [16]. A comparison of the Mini Nutrition Assessment Short Form (MNA-SF), the Malnutrition Universal Screening Tool, and the NRS-2002 in 215 hip fracture-operated elderly patients revealed these three screening tools to be adequate for assessing malnutrition, but only the MNA-SF could additionally predict readmissions and mortality [17]. In a study of 437 admitted patients with a proximal femoral fracture, a comparison of the MNA-SF and the Short Nutritional Assessment Questionnaire (SNAQ), a questionnaire consisting of three questions concerning weight loss, appetite, and the use of dietary supplements according to the guidelines for elderly patients with femoral fractures [18], revealed no benefits of the SNAQ over the MNA-SF as a screening tool for malnutrition [19]. However, all of the above questionnaires have one prominent disadvantage, that is, it is difficult to complete them when the elderly patients have difficulties in communication, such as in the case of those who are unconscious or under intubation. Furthermore, some important information may not be recollected by the patients [20]. In a review of 44 studies involving 26,281 patients, the prevalence of malnutrition was approximately $45.7 \%$ when different criteria such as body mass index (BMI), weight loss, or albumin concentration were considered; however, it was approximately $18.7 \%$ using the MNA [21]. Thus, researchers may prefer to use the data of a physical measurement or a biochemical profile than a questionnaire to serve as a screening tool for nutritional assessment. The Nutritional Risk Index (NRI), calculated based on the level of albumin, current body weight, and usual body weight, was proposed for the evaluation of malnutrition status [22]. However, the score is often difficult to obtain because half of the elderly patients cannot recollect their own usual body weight [20]. An updated form of the NRI, the geriatric nutritional risk index (GNRI), was proposed by Bouillanne et al. to evaluate 6-month midterm nutritional outcomes of hospitalized elderly patients in a rehabilitation unit [23]. By replacing the usual body weight in the formula by the ideal body weight [23], the GNRI acts as a simple screening tool to assess the nutrition-related risk of morbidity and mortality in hospitalized elderly patients $[24,25]$. The GNRI has been validated to have a strong correlation with the mid-upper arm muscle circumference, arm muscle area, and handgrip strength of hospitalized patients [26], preoperative sarcopenia status of cancer patients [27], and MNA score [28]. The GNRI was reported to be a strong prognostic factor to assess the outcomes of various clinical conditions, including chronic kidney disease [29], heart failure [30], chronic obstructive pulmonary disease [31], chronic hemodialysis [32], and certain malignancies [33,34]. Serum albumin level has been recognized as a strong indicator of patients' nutritional status. A low serum albumin level and total lymphocyte count at admission were significant predictors of one-year mortality in 174 elderly patients with intertrochanteric fractures [35]. A multiple logistic regression analysis in 127 patients who 
underwent surgery for femoral neck or trochanteric fractures revealed that the serum albumin level $(\mathrm{OR}=5.9, p=0.0004)$ and BMI $(\mathrm{OR}=1.2, p=0.0192)$ were significantly associated with mortality [36]. In a review of 19 studies involving 34,363 adults aged 74-85 years who underwent hip fracture surgery, the evaluation of predictive value of serum albumin level, total lymphocyte count at admission, and the MNA on patients' nutritional status demonstrated that a low serum albumin level is a sole indicator of increased risks of postoperative complications, in-hospital mortality, and total mortality [37]. However, serum albumin levels can be influenced by the hydration status, inflammatory processes, and impairments of hepatic or renal function [28]. The GNRI is a simple tool based on two main components: the serum albumin level and the ratio of present body weight to ideal body weight. In addition to the albumin level, a second parameter is included in the GNRI to increase its predictive value. It was shown in a 3 year observational study conducted in institutionalized elderly that the GNRI had a higher prognostic value for mortality than albumin alone [38]. The ratio of present body weight to ideal body weight, the second parameter of the GNRI, may reflect the long-term nutritional condition of patients. Notably, pneumonia (37\%) followed by acute coronary syndrome (31\%) and sepsis $(14 \%)$ are the most common causes of mortality in patients with hip fracture [39]. Death might be a consequence of nutrition-related complications [40,41]. Therefore, this study aimed to investigate whether the GNRI has a prognostic value that links the nutritional status and mortality outcomes in elderly patients who have previously undergone trauma with femoral fractures.

\section{Methods}

\subsection{Ethics Statement}

The study was approved before its implementation by the Institutional Review Board (IRB) of Chang Gung Memorial Hospital (approval number 202001446B0). Informed consent was waived according to the regulations of the IRB because the study was based on a retrospective analysis of registered data.

\subsection{Data Extraction}

Of the 39,135 enrolled patients with trauma-related injuries hospitalized for treatment between January 1, 2009 and December 31, 2019, 10,790 were elderly patients aged $\geq 65$ years. Among these elderly patients, 3737 patients sustained a trauma femoral fracture. All types of femoral fractures due to the trauma injury were included in this study. After excluding the patients who had no recorded data of albumin levels $(n=3016)$ and who had incomplete data of body weight or pre-existing comorbidities $(n=43), 678$ elderly patients with femoral fractures were enrolled in the study (Figure 1$)$. The study population was categorized into four nutritional risk groups: a major-risk group (GNRI < 82; group 1, $n=127$ ), moderate-risk group (GNRI 82 to $<92$; group 2, $n=179$ ), low-risk group (GNRI 92-98; group 3 , $n=123$ ), and no-risk group (GNRI > 98; group 4, $n=249$ ) according to the original description provided by Bouillanne et al. [23]. The following medical information of these patients was extracted from the Trauma Registry System of the hospital [42-44]: sex, age, BMI, albumin level on admission, preexisting comorbidities (diabetes mellitus (DM), hypertension (HTN), coronary artery disease, congestive heart failure, cerebral vascular accident (CVA), and end-stage renal disease), Glasgow coma scale (GCS) score, injury severity score (ISS), and in-hospital mortality. ISS was obtained from the sum of the squares of the three highest abbreviated injury scale scores of different body regions and represented the injury severity of the patients [45-47]. The GNRI was calculated as follows: $(1.489 \times$ albumin $(\mathrm{g} / \mathrm{dl})$ $+41.7 \times$ (body weight/ideal body weight)) originally proposed by Bouillanne et al. [23]. 


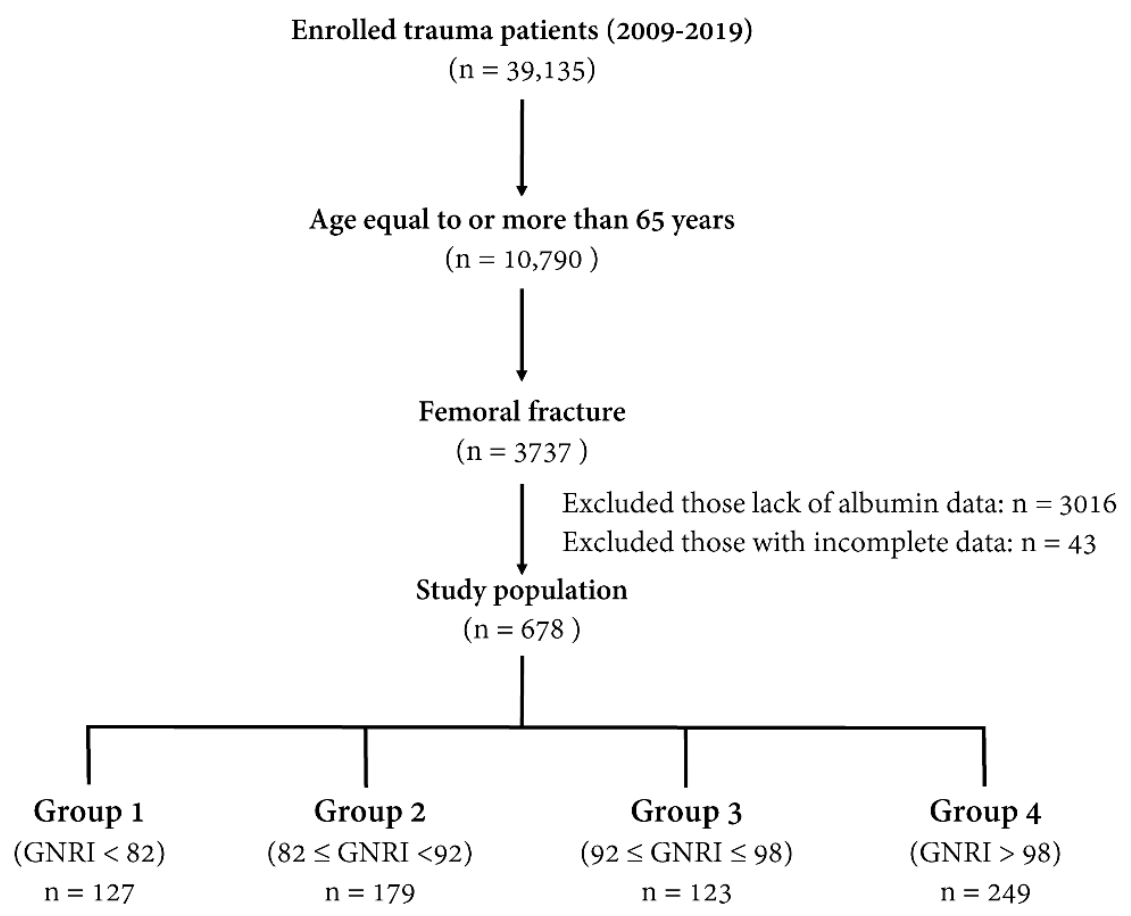

Figure 1. A flow chart illustrating the inclusion of elderly patients who had undergone trauma with femoral fractures, with the allocation of these patients into four nutritional risk groups.

\subsection{Statistical Analysis}

The data set for continuous variables was analyzed by the Kolmogorov-Smirnov test for distribution of normalization. Analysis of variance and the Mann-Whitney U-test were used to analyze normally and non-normally distributed continuous data, respectively, with Bonferroni post hoc correction. The results are expressed as mean \pm standard deviation or medians and interquartile ranges (IQR, Q1-Q3). Categorical data were compared using two-sided Fisher's exact or Pearson $\chi^{2}$ tests with the presentation of odds ratios (ORs) and 95\% confidence intervals (CIs). To minimize the confounding effects of sex, age, preexisting comorbidities, and injury severity of patients on outcome measurements, a logistic regression model was used to calculate the propensity scores with the above covariates and a 1:1 propensity score-matched patient cohort against the no-risk group (group 4) were created using the NCSS 10 software (NCSS statistical software, Kaysville, UT, USA) with the greedy method and a 0.2-caliper width. These matched patient cohorts were used to assess the impact of patients being in different nutritional risk groups on mortality outcomes in the no-risk group. In this study, the in-hospital mortality of patients was defined as the primary outcome and all statistical analyses were performed using Windows version 23.0 for SPSS (IBM Inc., Chicago, IL, USA). $p$ values $<0.05$ were considered statistically significant.

\section{Results}

\subsection{Patient and Injury Characteristics}

A comparison of the groups with different risks for malnutrition in the study population showed that there was a significantly higher percentage of male patients in group 1 than in group 4 (Table 1). The patients in groups 1-3 were significantly older and presented a significantly lower BMI and level of albumin than the patients in group 4 . There were significant intergroup differences in the prevalence of preexisting comorbidities of DM, HTN, and CVA. Additionally, there were significant intergroup differences in the GCS scores $(p=0.016)$, with a significantly lower GCS score in group 2 than in group 4 . When stratified by GCS scores (3-8, 9-12, or 13-15), significantly more patients had GCS scores of 9-12 in group 1 and group 2, but fewer patients had GCS scores of 13-15 in group 
2 than in group 4. There was no significant difference in the ISS among these groups of patients $(p=0.705)$ regardless of ISS stratification $(1-15,16-24$, and $\geq 25)$. Compared to the patients in group 4, a significantly higher mortality rate was found in those in group 1 (17.3\% vs. 3.6\% for group 1 vs. group 4 patients, respectively, $p<0.001)$, but not in those in group $2(6.7 \%)$ or group $3(2.4 \%)$.

Table 1. Patient and injury characteristics of the groups of patients with different risks of malnutrition.

\begin{tabular}{|c|c|c|c|c|c|}
\hline Variables & Group 1 & Group 2 & Group 3 & Group 4 & $p$ \\
\hline Range of GNRI & GNRI $<82$ & $82 \leq \mathrm{GNRI}<92$ & $92 \leq \mathrm{GNRI} \leq 98$ & GNRI $>98$ & \\
\hline Number of patients & $n=127$ & $n=179$ & $n=123$ & $n=249$ & \\
\hline Gender & & & & & 0.033 \\
\hline Male, $n(\%)$ & $53(41.7) *$ & $67(37.4)$ & $43(35.0)$ & $69(27.7)$ & \\
\hline Female, $n(\%)$ & $74(58.3) *$ & $112(62.6)$ & $80(65.0)$ & $180(72.3)$ & \\
\hline Age (years) & $81.8 \pm 7.3^{*}$ & $81.3 \pm 7.5^{*}$ & $78.9 \pm 6.9$ & $77.4 \pm 7.5$ & $<0.001$ \\
\hline BMI & $18.8 \pm 2.9 *$ & $21.9 \pm 2.8^{*}$ & $23.4 \pm 3.0^{*}$ & $26.6 \pm 3.9$ & $<0.001$ \\
\hline Albumin level (g/dL) & $2.6 \pm 0.5^{*}$ & $3.0 \pm 0.5^{*}$ & $3.3 \pm 0.5^{*}$ & $3.8 \pm 0.5$ & $<0.001$ \\
\hline \multicolumn{6}{|l|}{ Comorbidities } \\
\hline $\mathrm{DM}, n(\%)$ & $28(22.0)^{*}$ & $50(27.9) *$ & $46(37.4)$ & $110(44.2)$ & $<0.001$ \\
\hline HTN, $n(\%)$ & $67(52.8)$ * & $113(63.1)$ & $90(73.2)$ & $178(71.5)$ & 0.001 \\
\hline $\mathrm{CAD}, n(\%)$ & $16(12.6)$ & $18(10.1)$ & $20(16.3)$ & $37(14.9)$ & 0.374 \\
\hline $\mathrm{CHF}, n(\%)$ & $6(4.7)$ & $8(4.5)$ & $6(4.9)$ & $7(2.8)$ & 0.695 \\
\hline CVA, $n(\%)$ & $12(9.4)$ & $20(11.2)$ & $29(23.6)$ * & $27(10.8)$ & 0.002 \\
\hline ESRD, $n(\%)$ & $2(1.6)$ & $15(8.4)$ & $5(4.1)$ & $14(5.6)$ & 0.063 \\
\hline GCS, median (IQR) & $15(15-15)$ & $15(15-15)$ * & $15(15-15)$ & $15(15-15)$ & 0.016 \\
\hline $3-8$ & $1(0.8)$ & $6(3.4)$ & $1(0.8)$ & $4(1.6)$ & 0.265 \\
\hline $9-12$ & $8(6.3) *$ & $11(6.1) *$ & $3(2.4)$ & $1(0.4)$ & 0.002 \\
\hline $13-15$ & $118(92.9)$ & $162(90.5)^{*}$ & 119 (96.7) & $244(98)$ & 0.003 \\
\hline ISS, median (IQR) & $9(9-9)$ & $9(9-9)$ & $9(9-9)$ & $9(9-9)$ & 0.705 \\
\hline $1-15$ & $121(95.3)$ & $165(92.2)$ & $114(92.7)$ & $230(92.4)$ & 0.718 \\
\hline $16-24$ & $1(0.8)$ & $4(2.2)$ & $3(2.4)$ & $9(3.6)$ & 0.417 \\
\hline$\geq 25$ & $5(3.9)$ & $10(5.6)$ & $6(4.9)$ & $10(4.0)$ & 0.863 \\
\hline Mortality, $n(\%)$ & $22(17.3)^{*}$ & $12(6.7)$ & $3(2.4)$ & $9(3.6)$ & $<0.001$ \\
\hline
\end{tabular}

\subsection{Comparison of Propensity Score-Matched Patient Cohorts}

To minimize the confounding effects of sex, age, preexisting comorbidities, and injury severity of patients on outcome measurements, a 1:1 propensity score-matched patient cohort was created separately for the patients in groups 1-3 against those in group 4 (Supplemental Tables S1-S3). The selected pairs of propensity score-matched patient populations were those who did not present significant differences in sex, age, comorbidities, and ISS (Supplemental Tables S1-S3). As shown in Table 2, among the selected 75 pairs of patients, the patients in group 1 had significantly higher odds of mortality than those in group $4(\mathrm{OR}, 6.3 ; 95 \% \mathrm{CI}, 1.34-29.37 ; p=0.009)$. The patients in group 2 and group 3 did not have significantly different odds of mortality than those in group 4 among the selected 95 and 88 pairs of patients, respectively, in pairs of matched patient populations. 
Table 2. Comparison of the mortality outcomes in the propensity-score matched cohorts of the elderly patients with femoral fractures in group 1-3 vs. group 4 patients.

\begin{tabular}{ccccccccc}
\hline Groups of Comparison & $\begin{array}{c}\text { Numbers of } \\
\text { Pairs of Patients } \\
\boldsymbol{n}\end{array}$ & $\begin{array}{c}\text { Mortality of } \\
\text { Compared Group } \\
\boldsymbol{n} \mathbf{( \% )}\end{array}$ & $\begin{array}{c}\text { Mortality of } \\
\text { Group 4 } \\
\boldsymbol{( \% )}\end{array}$ & OR (95\% CI) & $\boldsymbol{p}$ \\
\hline Group 1 vs. Group 4 & $\mathbf{7 5}$ & $\mathbf{1 1}$ & $(14.7)$ & 2 & $(2.7)$ & 6.3 & $(1.34-29.37)$ & 0.009 \\
Group 2 vs. Group 4 & 95 & 7 & $(7.4)$ & 2 & $(2.1)$ & 3.7 & $(0.75-18.29)$ & 0.088 \\
Group 3 vs. Group 4 & 88 & 2 & $(2.3)$ & 5 & $(5.7)$ & 0.4 & $(0.07-2.05)$ & 0.247 \\
\hline
\end{tabular}

\section{Discussion}

The study results demonstrated that the GNRI helps classify malnutrition risk in elderly patients who had undergone trauma with femoral fractures. In elderly trauma patients with femoral fractures, the patients with GNRI $<82$ presented with a significant 6.3 -fold adjusted mortality risk compared with the patients in the no-risk group whose GNRI $>98$. The cutoff value was chosen according to the recommendations of Bouillanne et al., who determined four cutoff values for GNRI (GNRI < 82, GNRI 82 to $<92$, GNRI 92-98, and GNRI $>98$ ) to indicate the risk of malnutrition [23]. However, some different GNRI cutoff values have been reported for various diseases or clinical situations [48-50]. In patients undergoing hemodialysis, a GNRI $<100$ was significantly associated with mortality outcomes (hazard ratio 3.691; 95\% CI, 1.75-7.78; $p=0.001$ ) [51]. In patients undergoing pancreaticoduodenectomy, a GNRI < 94 was an independent predictor of surgical site infection (relative risk 1.73; 95\% CI, 1.23-2.43; $p<0.001$ ) [52]. For elderly patients with sepsis in the acute-care setting, a GNRI $<87$ has been proposed as the optimal cutoff indicating the requirement of nutritional support [53].

This study has some limitations and thus the results may be regarded as preliminary and require further validation. First, the retrospective design of this study may lead to a selection bias, especially considering that a large proportion of patients with femoral fractures did not have serum albumin data at admission. However, this also reflects that the assessment of the nutritional status is often neglected even for the patients with femoral fractures. Second, the trauma registry data only recorded in-hospital mortality but not data of 30 days or beyond; thus, the results may not reflect the full scope of the mortality associated with femoral fractures in the elderly. In addition, the patients declared dead on arrival in the emergency room were not included in the database, which may have led to a selection bias in the outcome measurement. Third, this study did not investigate interventions such as resuscitation, immobilization, and surgery conducted on patients; thus, the outcome measurements may be biased. Under these circumstances, we can only assume that the outcomes of the treatments were uniform across the study population. Fourth, a selection bias may occur because most patients with femoral fractures in the database did not have records of albumin levels and thus were excluded from the study; however, these data also reflected that the nutrition status was generally neglected by the orthopedists and trauma surgeons. Finally, the population included in this study was limited to that of a single urban trauma center, and the results may not be applicable to a wider population.

\section{Conclusions}

This preliminary report suggests that the GNRI may be used as a screening tool to identify patients with malnutrition at a high risk of mortality among elderly patients with femoral fractures. A prospective study is needed to validate the finding.

Supplementary Materials: The following are available online at http://www.mdpi.com/1660-4601/17/23/8920/s1, Table S1: Comparison of the mortality outcomes in the propensity-score matched cohorts of the elderly patients with femoral fractures in group 1 vs. group 4 patients; Table S2: Comparison of the mortality outcomes in the propensity-score matched cohorts of the elderly patients with femoral fractures in group 2 vs. group 4 patients; Table S3: Comparison of the mortality outcomes in the propensity-score matched cohorts of the elderly patients with femoral fractures in group 3 vs. group 4 patients.

Author Contributions: W.-T.S. wrote the manuscript; S.-C.W. reviewed the literature; C.-Y.H. \& S.-E.C. helped to maintain the integrity of the registered data; C.-H.T. contributed to the editing of tables; C.L. proofread the 
manuscript; S.-Y.H. performed the statistical analyses; C.-H.H. designed the study and contributed to the analysis and interpretation of data. All authors have read and agreed to the published version of the manuscript.

Funding: This research was supported by a grant from CMRPG8K1611 to Wei-Ti Su.

Acknowledgments: We would appreciate the assistance provided by Biostatistics Center, Kaohsiung Chang Gung Memorial Hospital for the statistical analyses.

Conflicts of Interest: The authors declare no conflict of interest.

\section{References}

1. Lindner, T.; Kanakaris, N.K.; Marx, B.; Cockbain, A.; Kontakis, G.; Giannoudis, P.V. Fractures of the hip and osteoporosis: The role of bone substitutes. J. Bone Joint Surg. Br. 2009, 91, 294-303. [CrossRef]

2. Cauley, J.A. Osteoporosis: Fracture epidemiology update 2016. Curr. Opin. Rheumatol. 2017, 29, 150-156. [CrossRef]

3. Chang, M.W.; Liu, H.T.; Huang, C.Y.; Chien, P.C.; Hsieh, H.Y.; Hsieh, C.H. Location of femoral fractures in patients with different weight classes in fall and motorcycle accidents: A Retrospective cross-sectional analysis. Int. J. Environ. Res. Public Health 2018, 15, 1082. [CrossRef]

4. Chen, C.C.; Rau, C.S.; Wu, S.C.; Kuo, P.J.; Chen, Y.C.; Hsieh, H.Y.; Hsieh, C.H. Association of osteoporosis self-assessment tool for Asians (OSTA) score with clinical presentation and expenditure in hospitalized trauma patients with femoral fractures. Int. J. Environ. Res. Public Health 2016, 13, 995. [CrossRef]

5. Rau, C.S.; Wu, S.C.; Chen, Y.C.; Chien, P.C.; Hsieh, H.Y.; Kuo, P.J.; Hsieh, C.H. Mortality Rate associated with admission hyperglycemia in traumatic femoral fracture patients is greater than non-diabetic normoglycemic patients but not diabetic normoglycemic patients. Int. J. Environ. Res. Public Health 2017, 15, 28. [CrossRef]

6. Rau, C.S.; Wu, S.C.; Kuo, P.J.; Chen, Y.C.; Chien, P.C.; Hsieh, H.Y.; Hsieh, C.H. Epidemiology of bone fracture in female trauma patients based on risks of osteoporosis assessed using the osteoporosis self-assessment tool for Asians score. Int. J. Environ. Res. Public Health 2017, 14, 1380. [CrossRef]

7. Tang, C.E.; Liu, H.T.; Kuo, P.J.; Chen, Y.C.; Hsu, S.Y.; Lin, C.C.; Hsieh, C.H. Impact of sexual dimorphism on trauma patterns and clinical outcomes of patients with a high-risk score of the osteoporosis self-assessment tool for Asians: A propensity score-matched analysis. Int. J. Environ. Res. Public Health 2018, 15, 418. [CrossRef]

8. Barker, L.A.; Gout, B.S.; Crowe, T.C. Hospital malnutrition: Prevalence, identification and impact on patients and the healthcare system. Int. J. Environ. Res. Public Health 2011, 8, 514-527. [CrossRef]

9. Gavazzi, G.; Krause, K.H. Ageing and infection. Lancet Infect. Dis. 2002, 2, 659-666. [CrossRef]

10. Kuzu, M.A.; Terzioğlu, H.; Genç, V.; Erkek, A.B.; Ozban, M.; Sonyürek, P.; Elhan, A.H.; Torun, N. Preoperative nutritional risk assessment in predicting postoperative outcome in patients undergoing major surgery. World J. Surg. 2006, 30, 378-390. [CrossRef]

11. Schaible, U.E.; Kaufmann, S.H. Malnutrition and infection: Complex mechanisms and global impacts. PLoS Med. 2007, 4, e115. [CrossRef] [PubMed]

12. Van Wissen, J.; van Stijn, M.F.; Doodeman, H.J.; Houdijk, A.P. Mini nutritional assessment and mortality after hip fracture surgery in the elderly. J. Nutr. Health Aging 2016, 20, 964-968. [CrossRef]

13. Zanetti, M.; Cappellari, G.G.; Ratti, C.; Ceschia, G.; Murena, L.; de Colle, P.; Barazzoni, R. Poor nutritional status but not cognitive or functional impairment per se independently predict 1 year mortality in elderly patients with hip-fracture. Clin. Nutr. 2019, 38, 1607-1612. [CrossRef]

14. Green, S.M.; Watson, R. Nutritional screening and assessment tools for older adults: Literature review. J. Adv. Nurs. 2006, 54, 477-490. [CrossRef]

15. Van Bokhorst-de van der Schueren, M.A.; Guaitoli, P.R.; Jansma, E.P.; de Vet, H.C. Nutrition screening tools: Does one size fit all? A systematic review of screening tools for the hospital setting. Clin. Nutr. 2014, 33, 39-58. [CrossRef]

16. Goost, H.; Vidakovic, E.; Deborre, C.; Randau, T.; Wirtz, D.C.; Burger, C.; Koch, E.; Kabir, K. Malnutrition in geriatric trauma patients: Screening methods in comparison. Technol. Health Care Off. J. Eur. Soc. Eng. Med. 2016, 24, 225-239. [CrossRef]

17. Koren-Hakim, T.; Weiss, A.; Hershkovitz, A.; Otzrateni, I.; Anbar, R.; Nevo, R.F.G.; Schlesinger, A.; Frishman, S.; Salai, M.; Beloosesky, Y. Comparing the adequacy of the MNA-SF, NRS-2002 and MUST nutritional tools in assessing malnutrition in hip fracture operated elderly patients. Clin. Nutr. 2016, 35, 1053-1058. [CrossRef] 
18. DICA. Dutch Hip Fracture Audit DHFA. Available online: https://dica.nl/dhfa/home (accessed on 30 September 2020).

19. Van der Sijp, M.P.L.; van Eijk, M.; Krijnen, P.; Schipper, I.B.; Achterberg, W.P.; Niggebrugge, A.H.P. Screening for malnutrition in patients admitted to the hospital with a proximal femoral fracture. Injury 2018, 49, 2239-2243. [CrossRef]

20. Kuczmarski, M.F.; Kuczmarski, R.J.; Najjar, M. Effects of age on validity of self-reported height, weight, and body mass index: Findings from the Third National Health and Nutrition Examination Survey, 1988-1994. J. Am. Diet. Assoc. 2001, 101, 28-34. [CrossRef]

21. Malafarina, V.; Reginster, J.Y.; Cabrerizo, S.; Bruyère, O.; Kanis, J.A.; Martinez, J.A.; Zulet, M.A. Nutritional status and nutritional treatment are related to outcomes and mortality in older adults with hip fracture. Nutrients 2018, 10, 555. [CrossRef]

22. Buzby, G.P.; Knox, L.S.; Crosby, L.O.; Eisenberg, J.M.; Haakenson, C.M.; McNeal, G.E.; Page, C.P.; Peterson, O.L.; Reinhardt, G.F.; Williford, W.O. Study protocol: A randomized clinical trial of total parenteral nutrition in malnourished surgical patients. Am. J. Clin. Nutr. 1988, 47, 366-381. [CrossRef] [PubMed]

23. Bouillanne, O.; Morineau, G.; Dupont, C.; Coulombel, I.; Vincent, J.P.; Nicolis, I.; Benazeth, S.; Cynober, L.; Aussel, C. Geriatric nutritional risk index: A new index for evaluating at-risk elderly medical patients. Am. J. Clin. Nutr. 2005, 82, 777-783. [CrossRef]

24. Abd-El-Gawad, W.M.; Abou-Hashem, R.M.; El Maraghy, M.O.; Amin, G.E. The validity of Geriatric Nutrition Risk Index: Simple tool for prediction of nutritional-related complication of hospitalized elderly patients. Comparison with Mini Nutritional Assessment. Clin. Nutr. 2014, 33, 1108-1116. [CrossRef]

25. Cereda, E.; Pedrolli, C.; Zagami, A.; Vanotti, A.; Piffer, S.; Faliva, M.; Rondanelli, M.; Caccialanza, R. Nutritional risk, functional status and mortality in newly institutionalised elderly. Br. J. Nutr. 2013, 110, 1903-1909. [CrossRef]

26. Cereda, E.; Vanotti, A. The new Geriatric Nutritional Risk Index is a good predictor of muscle dysfunction in institutionalized older patients. Clin. Nutr. 2007, 26, 78-83. [CrossRef]

27. Han, J.Y.; Lee, K.H.; Kim, S.W.; Min, Y.J.; Cho, E.; Lee, Y.; Lee, S.H.; Kim, H.Y.; Lee, G.K.; Nam, B.H.; et al. A Phase II study of poziotinib in patients with Epidermal Growth Factor Receptor (EGFR)-mutant lung adenocarcinoma who have acquired resistance to EGFR-tyrosine kinase inhibitors. Cancer Res. Treat. Off. J. Korean Cancer Assoc. 2017, 49, 10-19. [CrossRef]

28. Alert, P.D.; Villarroel, R.M.; Formiga, F.; Casas, N.V.; Farré, C.V. Assessing risk screening methods of malnutrition in geriatric patients: Mini Nutritional Assessment (MNA) versus Geriatric Nutritional Risk Index (GNRI). Nutr. Hosp. 2012, 27, 590-598. [CrossRef]

29. Kuo, I.C.; Huang, J.C.; Wu, P.Y.; Chen, S.C.; Chang, J.M.; Chen, H.C. A low geriatric nutrition risk index is associated with progression to dialysis in patients with chronic kidney disease. Nutrients 2017, 9, 1228. [CrossRef]

30. Nishi, I.; Seo, Y.; Hamada-Harimura, Y.; Yamamoto, M.; Ishizu, T.; Sugano, A.; Sato, K.; Sai, S.; Obara, K.; Suzuki, S.; et al. Geriatric nutritional risk index predicts all-cause deaths in heart failure with preserved ejection fraction. ESC Heart Fail. 2019, 6, 396-405. [CrossRef]

31. Matsumura, T.; Mitani, Y.; Oki, Y.; Fujimoto, Y.; Ohira, M.; Kaneko, H.; Kawashima, T.; Nishio, M.; Ishikawa, A. Comparison of Geriatric Nutritional Risk Index scores on physical performance among elderly patients with chronic obstructive pulmonary disease. Heart Lung 2015, 44, 534-538. [CrossRef]

32. Xiong, J.; Wang, M.; Zhang, Y.; Nie, L.; He, T.; Wang, Y.; Huang, Y.; Feng, B.; Zhang, J.; Zhao, J. Association of Geriatric Nutritional Risk Index with mortality in hemodialysis patients: A meta-analysis of cohort studies. Kidney Blood Press. Res. 2018, 43, 1878-1889. [CrossRef]

33. Lidoriki, I.; Schizas, D.; Frountzas, M.; Machairas, N.; Prodromidou, A.; Kapelouzou, A.; Karavokyros, I.; Pikoulis, E.; Kales, S.N.; Liakakos, T. GNRI as a prognostic factor for outcomes in cancer patients: A systematic review of the literature. Nutr. Cancer 2020, 1-13. [CrossRef]

34. Lv, G.Y.; An, L.; Sun, D.W. Geriatric Nutritional Risk Index predicts adverse outcomes in human malignancy: A meta-analysis. Dis. Markers 2019, 2019, 4796598. [CrossRef]

35. Lu, J.; Chen, Y.Y.; Zhang, L.; Li, Y.G.; Wang, C. Laboratory nutritional parameters predict one-year mortality in elderly patients with intertrochanteric fracture. Asia Pac. J. Clin. Nutr. 2016, 25, 457-463. [CrossRef]

36. Miyanishi, K.; Jingushi, S.; Torisu, T. Mortality after hip fracture in Japan: The role of nutritional status. J. Orthop. Surg. 2010, 18, 265-270. [CrossRef] 
37. Li, S.; Zhang, J.; Zheng, H.; Wang, X.; Liu, Z.; Sun, T. Prognostic role of serum albumin, total lymphocyte count, and mini nutritional assessment on outcomes after geriatric hip fracture surgery: A meta-analysis and systematic review. J. Arthroplasty 2019, 34, 1287-1296. [CrossRef]

38. Cereda, E.; Zagami, A.; Vanotti, A.; Piffer, S.; Pedrolli, C. Geriatric Nutritional Risk Index and overall-cause mortality prediction in institutionalised elderly: A 3-year survival analysis. Clin. Nutr. 2008, 27, 717-723. [CrossRef]

39. Khan, S.K.; Rushton, S.P.; Shields, D.W.; Corsar, K.G.; Refaie, R.; Gray, A.C.; Deehan, D.J. The risk of cardiorespiratory deaths persists beyond 30 days after proximal femoral fracture surgery. Injury 2015, 46, 358-362. [CrossRef]

40. Artinyan, A.; Orcutt, S.T.; Anaya, D.A.; Richardson, P.; Chen, G.J.; Berger, D.H. Infectious postoperative complications decrease long-term survival in patients undergoing curative surgery for colorectal cancer: A study of 12,075 patients. Ann. Surg. 2015, 261,497-505. [CrossRef]

41. Nathan, H.; Yin, H.; Wong, S.L. Postoperative complications and long-term survival after complex cancer resection. Ann. Surg. Oncol. 2017, 24, 638-644. [CrossRef]

42. Hsieh, C.H.; Hsu, S.Y.; Hsieh, H.Y.; Chen, Y.C. Differences between the sexes in motorcycle-related injuries and fatalities at a Taiwanese level I trauma center. Biomed. J. 2017, 40, 113-120. [CrossRef]

43. Hsieh, C.H.; Liu, H.T.; Hsu, S.Y.; Hsieh, H.Y.; Chen, Y.C. Motorcycle-related hospitalizations of the elderly. Biomed. J. 2017, 40, 121-128. [CrossRef]

44. Hsieh, C.H.; Chen, Y.C.; Hsu, S.Y.; Hsieh, H.Y.; Chien, P.C. Defining polytrauma by abbreviated injury scale $>/=3$ for a least two body regions is insufficient in terms of short-term outcome: A cross-sectional study at a level I trauma center. Biomed. J. 2018, 41, 321-327. [CrossRef]

45. Dong, X.R. Analysis of patients of multiple injuries with AIS-ISS and its clinical significance in the evaluation of the emergency managements. Zhonghua Wai Ke Za Zhi 1993, 31, 301-302.

46. Hsu, S.Y.; Wu, S.C.; Rau, C.S.; Hsieh, T.M.; Liu, H.T.; Huang, C.Y.; Chou, S.E.; Su, W.T.; Hsieh, C.H. Impact of adapting the Abbreviated Injury Scale (AIS)-2005 from AIS-1998 on injury severity scores and clinical outcome. Int. J. Env. Res. Public Health 2019, 16, 5033. [CrossRef]

47. Rau, C.S.; Wu, S.C.; Kuo, P.J.; Chen, Y.C.; Chien, P.C.; Hsieh, H.Y.; Hsieh, C.H. Same Abbreviated injury scale values may be associated with different risks to mortality in trauma patients: A Cross-sectional retrospective study based on the trauma registry system in a level i trauma center. Int. J. Environ. Res. Public Health 2017, 14, 1552. [CrossRef]

48. Takahashi, H.; Ito, Y.; Ishii, H.; Aoyama, T.; Kamoi, D.; Kasuga, H.; Yasuda, K.; Maruyama, S.; Matsuo, S.; Murohara, T.; et al. Geriatric nutritional risk index accurately predicts cardiovascular mortality in incident hemodialysis patients. J. Cardiol. 2014, 64, 32-36. [CrossRef]

49. Beberashvili, I.; Azar, A.; Sinuani, I.; Kadoshi, H.; Shapiro, G.; Feldman, L.; Averbukh, Z.; Weissgarten, J. Comparison analysis of nutritional scores for serial monitoring of nutritional status in hemodialysis patients. Clin. J. Am. Soc. Nephrol. CJASN 2013, 8, 443-451. [CrossRef]

50. Zhang, Y.; Zhang, Z.; Yu, Q.; Yuan, W. Value of the Geriatric Nutritional Risk Index in predicting mortality of elderly patients undergoing hemodialysis. Zhonghua Yi Xue Za Zhi 2015, 95, 3741-3745.

51. Edalat-Nejad, M.; Zameni, F.; Qlich-Khani, M.; Salehi, F. Geriatric nutritional risk index: A mortality predictor in hemodialysis patients. Saudi J. Kidney Dis. Transplant. 2015, 26, 302-308. [CrossRef]

52. Funamizu, N.; Nakabayashi, Y.; Iida, T.; Kurihara, K. Geriatric nutritional risk index predicts surgical site infection after pancreaticoduodenectomy. Mol. Clin. Oncol. 2018, 9, 274-278. [CrossRef]

53. Lee, J.S.; Choi, H.S.; Ko, Y.G.; Yun, D.H. Performance of the Geriatric Nutritional Risk Index in predicting 28-day hospital mortality in older adult patients with sepsis. Clin. Nutr. 2013, 32, 843-848. [CrossRef]

Publisher's Note: MDPI stays neutral with regard to jurisdictional claims in published maps and institutional affiliations. 